International Journal of Information Technology Convergence and Services (IJITCS) Vol.1, No.3, June 2011

\title{
ADAPTIVE CONTROL AND SYNCHRONIZATION OF HyPERCHAOTIC CHEN SYSTEM
}

\author{
Sundarapandian Vaidyanathan ${ }^{1}$ \\ ${ }^{1}$ Research and Development Centre, Vel Tech Dr. RR \& Dr. SR Technical University \\ Avadi, Chennai-600 062, Tamil Nadu, INDIA \\ sundarvtu@gmail. com
}

\section{ABSTRACT}

The hyperchaotic Newton-Leipnik system (Ghosh and Bhattacharya, 2010) is one of the recently discovered four-dimensional hyperchaotic systems. This paper investigates the adaptive control and synchronization of hyperchaotic Newton-Leipnik system with unknown parameters. First, adaptive control laws are designed to stabilize the hyperchaotic Newton-Leipnik system to its unstable equilibrium at the origin based on the adaptive control theory and Lyapunov stability theory. Then adaptive control laws are derived to achieve global chaos synchronization of identical hyperchaotic Newton-Leipnik systems with unknown parameters. Numerical simulations are presented to demonstrate the effectiveness of the proposed adaptive stabilization and synchronization schemes.

\section{KEYWORDS}

Adaptive Control, Stabilization, Chaos Synchronization, Hyperchaos, Hyperchaotic Newton-Leipnik System.

\section{INTRODUCTION}

Chaotic systems are dynamical systems that are highly sensitive to initial conditions. The sensitive nature of chaotic systems is commonly called as the butterfly effect [1]. Since chaos phenomenon in weather models was first observed by Lorenz in 1961, a large number of chaos phenomena and chaos behaviour have been discovered in physical, social, economical, biological and electrical systems.

The control of chaotic systems is to design state feedback control laws that stabilizes the chaotic systems around the unstable equilibrium points. Active control technique is used when the system parameters are known and adaptive control technique is used when the system parameters are unknown [2-4].

Synchronization of chaotic systems is a phenomenon that may occur when two or more chaotic oscillators are coupled or when a chaotic oscillator drives another chaotic oscillator. Because of the butterfly effect, which causes the exponential divergence of the trajectories of two identical chaotic systems started with nearly the same initial conditions, synchronizing two chaotic systems is seemingly a very challenging problem in the chaos literature [5-16].

In 1990, Pecora and Carroll [5] introduced a method to synchronize two identical chaotic systems and showed that it was possible for some chaotic systems to be completely synchronized. From then on, chaos synchronization has been widely explored in a variety of fields including physical systems [6], chemical systems [7], ecological systems [8], secure communications [9-10], etc. 
In most of the chaos synchronization approaches, the master-slave or drive-response formalism has been used. If a particular chaotic system is called the master or drive system and another chaotic system is called the slave or response system, then the idea of synchronization is to use the output of the master system to control the slave system so that the output of the slave system tracks the output of the master system asymptotically.

Since the seminal work by Pecora and Carroll [5], a variety of impressive approaches have been proposed for the synchronization of chaotic systems such as the sampled-data feedback synchronization method [11], OGY method [12], time-delay feedback method [13], backstepping method [14], adaptive design method [15], sliding mode control method [16], etc.

This paper is organized as follows. In Section 2, we derive results for the adaptive control of hyperchaotic Newton-Leipnik system (Ghosh and Bhattacharya, [17], 2010) with unknown parameters. In Section 3, we derive results for the adaptive synchronization of hyperchaotic Newton-Leipnik systems with unknown parameters. Section 4 contains the conclusions.

\section{AdAPTIVE CONTROL OF HyPERChaOtic NeWTON-LEIPNIK SYSTEM}

\subsection{Theoretical Results}

The hyperchaotic Newton-Leipnik system (Ghosh and Bhattacharya, [17], 2010) is one of the recently discovered four-dimensional hyperchaotic systems. The hyperchaotic Newton-Leipnik dynamics is described by

$$
\begin{aligned}
& \dot{x}_{1}=-a x_{1}+x_{2}+10 x_{2} x_{3}+x_{4} \\
& \dot{x}_{2}=-x_{1}-0.4 x_{2}+5 x_{1} x_{3} \\
& \dot{x}_{3}=b x_{3}-5 x_{1} x_{2} \\
& \dot{x}_{4}=-c x_{1} x_{3}+d x_{4}
\end{aligned}
$$

where $x_{i}(i=1,2,3,4)$ are the state variables and $a, b, c, d$ are positive constants.
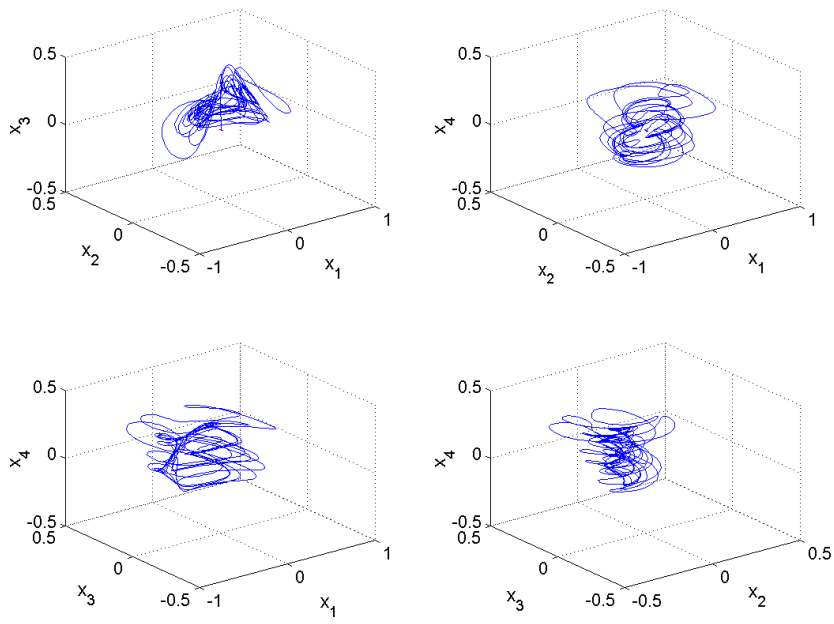

Figure 1. State Orbits of the Hyperchaotic Newton-Leipnik System 
International Journal of Information Technology Convergence and Services (IJITCS) Vol.1, No.3, June 2011

The system (1) is hyperchaotic when the parameter values are taken as

$$
a=0.4, \quad b=0.175, c=0.8 \text { and } d=0.01 .
$$

The hyperchaotic state portrait of the system (1) is described in Figure 1.

When the parameter values are taken as in (2), the system (1) is hyperchaotic and the system linearization matrix at the equilibrium point $E_{0}=(0,0,0,0)$ is given by

$$
A=\left[\begin{array}{cccc}
-0.4 & 1 & 0 & 1 \\
-1 & -0.4 & 0 & 0 \\
0 & 0 & 0.175 & 0 \\
0 & 0 & 0 & 0.01
\end{array}\right]
$$

which has the eigenvalues

$$
\lambda_{1}=-0.4+i, \lambda_{2}=-0.4-i, \lambda_{3}=0.01 \text { and } \lambda_{4}=0.175
$$

Since $\lambda_{3}$ and $\lambda_{4}$ are eigenvalues with positive real part, it is immediate from Lyapunov stability theory [18] that the system (1) is unstable at the equilibrium point $E_{0}=(0,0,0,0)$.

In this section, we design adaptive control law for globally stabilizing the hyperchaotic system (1) when the parameter values are unknown.

Thus, we consider the controlled hyperchaotic Newton-Leipnik system as follows.

$$
\begin{aligned}
& \dot{x}_{1}=-a x_{1}+x_{2}+10 x_{2} x_{3}+x_{4}+u_{1} \\
& \dot{x}_{2}=-x_{1}-0.4 x_{2}+5 x_{1} x_{3}+u_{2} \\
& \dot{x}_{3}=b x_{3}-5 x_{1} x_{2}+u_{3} \\
& \dot{x}_{4}=-c x_{1} x_{3}+d x_{4}+u_{4}
\end{aligned}
$$

where $u_{1}, u_{2}, u_{3}$ and $u_{4}$ are feedback controllers to be designed using the states and estimates of the unknown parameters of the system.

In order to ensure that the controlled system (3) globally converges to the origin asymptotically, we consider the following adaptive control functions

$$
\begin{aligned}
& u_{1}=\hat{a} x_{1}-x_{2}-10 x_{2} x_{3}-x_{4}-k_{1} x_{1} \\
& u_{2}=x_{1}+0.4 x_{2}-5 x_{1} x_{3}-k_{2} x_{2} \\
& u_{3}=-\hat{b} x_{3}+5 x_{1} x_{2}-k_{3} x_{3} \\
& u_{4}=\hat{c} x_{1} x_{3}-k_{4} x_{4}
\end{aligned}
$$

where $\hat{a}, \hat{b}, \hat{c}$ and $\hat{d}$ are estimates of the parameters $a, b, c$ and $d$, respectively, and $k_{i},(i=1,2,3,4)$ are positive constants. 
International Journal of Information Technology Convergence and Services (IJITCS) Vol.1, No.3, June 2011

Substituting the control law (4) into the hyperchaotic Newton-Leipnik dynamics (1), we obtain

$$
\begin{aligned}
& \dot{x}_{1}=-(a-\hat{a}) x_{1}-k_{1} x_{1} \\
& \dot{x}_{2}=-k_{2} x_{2} \\
& \dot{x}_{3}=(b-\hat{b}) x_{3}-k_{3} x_{3} \\
& \dot{x}_{4}=-(c-\hat{c}) x_{1} x_{3}+(d-\hat{d}) x_{4}-k_{4} x_{4}
\end{aligned}
$$

Let us now define the parameter errors as

$$
e_{a}=a-\hat{a}, \quad e_{b}=b-\hat{b}, \quad e_{c}=c-\hat{c} \text { and } e_{d}=d-\hat{d} .
$$

Using (6), the closed-loop dynamics (5) can be written compactly as

$$
\begin{aligned}
& \dot{x}_{1}=-e_{a} x_{1}-k_{1} x_{1} \\
& \dot{x}_{2}=-k_{2} x_{2} \\
& \dot{x}_{3}=e_{b} x_{3}-k_{3} x_{3} \\
& \dot{x}_{4}=-e_{c} x_{1} x_{3}+e_{d} x_{4}-k_{4} x_{4}
\end{aligned}
$$

For the derivation of the update law for adjusting the parameter estimates $\hat{a}, \hat{b}, \hat{c}, \hat{d}, \hat{\varepsilon}, \hat{f}$, the Lyapunov approach is used.

Consider the quadratic Lyapunov function

$$
V\left(x_{1}, x_{2}, x_{3}, x_{4}, e_{a}, e_{b}, e_{c}, e_{d}\right)=\frac{1}{2}\left(x_{1}^{2}+x_{2}^{2}+x_{3}^{2}+x_{4}^{2}+e_{a}^{2}+e_{b}^{2}+e_{c}^{2}+e_{d}^{2}\right)
$$

which is a positive definite function on $R^{8}$.

Note also that

$$
\begin{aligned}
& \dot{e}_{a}=-\dot{\hat{a}} \\
& \dot{e}_{b}=-\dot{\hat{b}} \\
& \dot{e}_{c}=-\dot{\hat{c}} \\
& \dot{e}_{d}=-\dot{\hat{d}}
\end{aligned}
$$

Differentiating $V$ along the trajectories of (7) and using (9), we obtain

$$
\begin{aligned}
\dot{V}= & -k_{1} x_{1}^{2}-k_{2} x_{2}^{2}-k_{3} x_{3}^{2}-k_{4} x_{4}^{2}+e_{a}\left[-x_{1}^{2}-\dot{\hat{a}}\right]+e_{b}\left[x_{3}^{2}-\dot{\hat{b}}\right] \\
& +e_{c}\left[-x_{1} x_{3} x_{4}-\dot{\hat{c}}\right]+e_{d}\left[x_{4}^{2}-\dot{\hat{d}}\right]
\end{aligned}
$$


In view of Eq. (10), the estimated parameters are updated by the following law:

$$
\begin{aligned}
& \dot{\hat{a}}=-x_{1}^{2}+k_{5} e_{a} \\
& \dot{\hat{b}}=x_{3}^{2}+k_{6} e_{b} \\
& \dot{\hat{c}}=-x_{1} x_{3} x_{4}+k_{7} e_{c} \\
& \dot{\hat{d}}=x_{4}^{2}+k_{8} e_{d}
\end{aligned}
$$

where $k_{5}, k_{6}, k_{7}$ and $k_{8}$ are positive constants.

Substituting (11) into (10), we get

$$
\dot{V}=-k_{1} x_{1}^{2}-k_{2} x_{2}^{2}-k_{3} x_{3}^{2}-k_{4} x_{4}^{2}-k_{5} e_{a}^{2}-k_{6} e_{b}^{2}-k_{7} e_{c}^{2}-k_{8} e_{d}^{2}
$$

which is a negative definite function on $R^{8}$.

Thus, by Lyapunov stability theory [18], we obtain the following result.

Theorem 1. The hyperchaotic Newton-Leipnik system (1) with unknown parameters is globally and exponentially stabilized for all initial conditions $x(0) \in R^{4}$ by the adaptive control law (4), where the update law for the parameters is given by $(11)$ and $k_{i},(i=1, \ldots, 8)$ are positive constants.

\subsection{Numerical Results}

For the numerical simulations, the fourth order Runge-Kutta method is used to solve the hyperchaotic system (1) with the adaptive control law (4) and the parameter update law (11).

The parameters of the hyperchaotic Newton-Leipnik system (1) are selected as

$$
a=0.4, b=0.175, c=0.8 \text { and } d=0.01 \text {. }
$$

For the adaptive and update laws, we take $k_{i}=2, \quad(i=1,2, \ldots, 8)$.

Suppose that the initial values of the estimated parameters are

$$
\hat{a}(0)=3, \hat{b}(0)=5, \quad \hat{c}(0)=8 \text { and } \hat{f}(0)=7 .
$$

The initial values of the hyperchaotic Newton-Leipnik system (1) are taken as $x(0)=(9,16,22,30)$.

When the adaptive control law (4) and the parameter update law (11) are used, the controlled hyperchaotic Newton-Leipnik system converges to the equilibrium $E_{0}=(0,0,0,0)$ exponentially as shown in Figure 2 . The parameter estimates are shown in Figure 3 . 
International Journal of Information Technology Convergence and Services (IJTTCS) Vol.1, No.3, June 2011
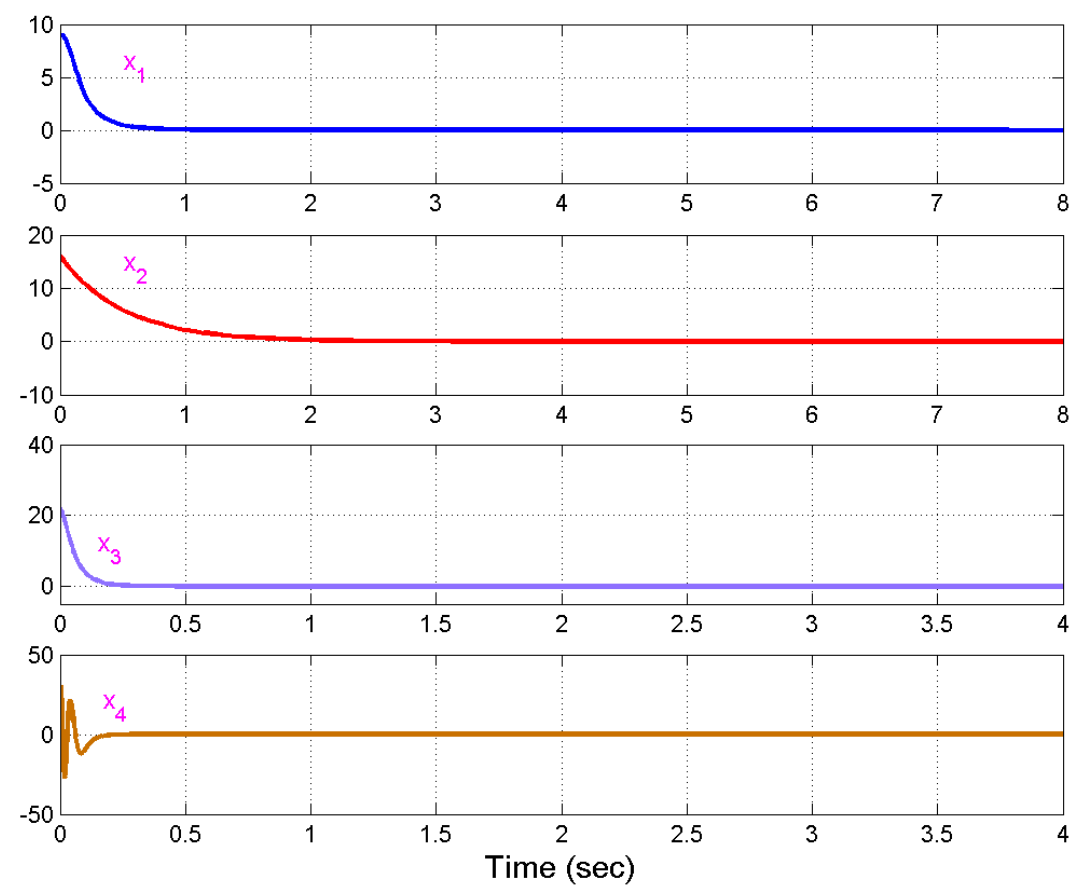

Figure 2. Time Responses of the Controlled Hyperchaotic Newton-Leipnik System

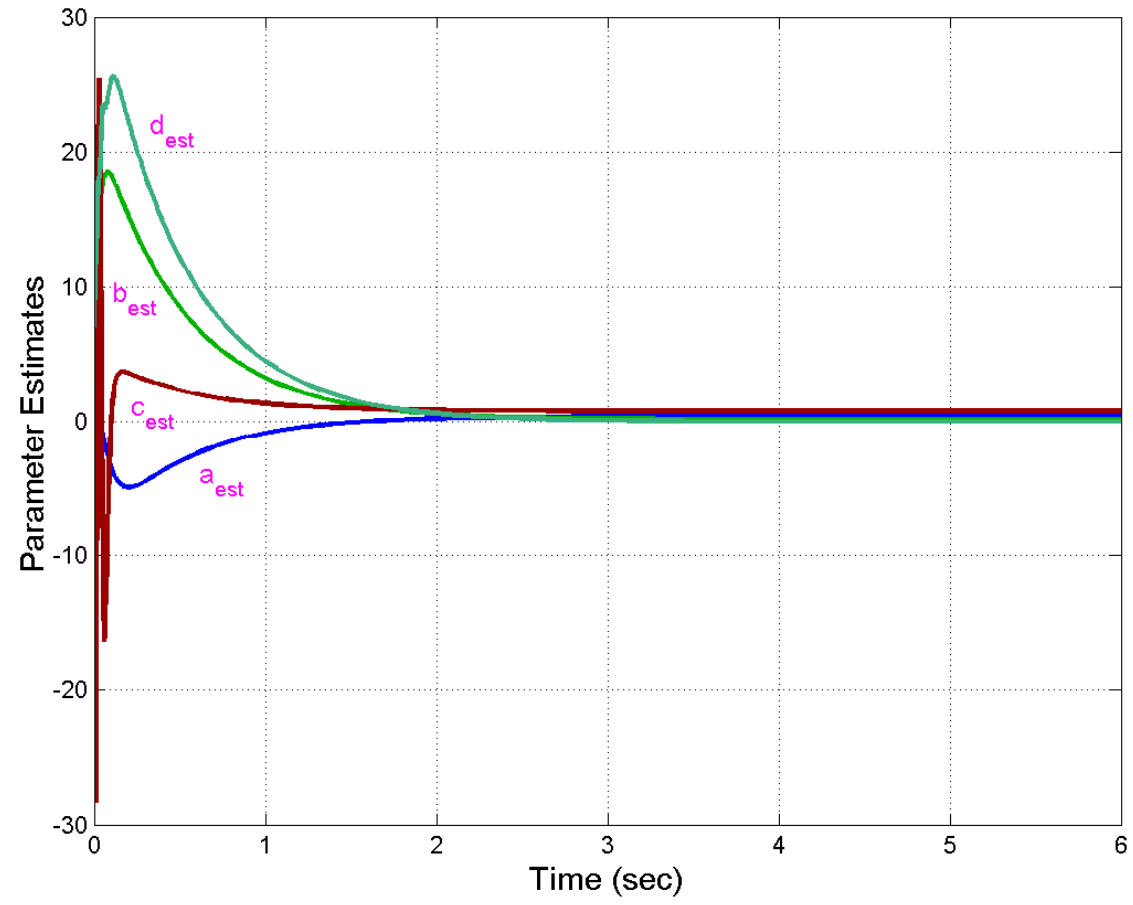

Figure 3. Parameter Estimates $\hat{a}(t), \hat{b}(t), \hat{c}(t), \hat{d}(t)$ 
International Journal of Information Technology Convergence and Services (IJITCS) Vol.1, No.3, June 2011

\section{AdAPTIVE Synchronization OF IDENTICAL HyPERCHAOTIC NEWTON-LEIPNIK SYSTEMS}

\subsection{Theoretical Results}

In this section, we discuss the adaptive synchronization of identical hyperchaotic NewtonLeipnik systems (Ghosh and Bhattacharya, [17], 2010) with unknown parameters.

As the master system, we consider the hyperchaotic Newton-Leipnik dynamics described by

$$
\begin{aligned}
& \dot{x}_{1}=-a x_{1}+x_{2}+10 x_{2} x_{3}+x_{4} \\
& \dot{x}_{2}=-x_{1}-0.4 x_{2}+5 x_{1} x_{3} \\
& \dot{x}_{3}=b x_{3}-5 x_{1} x_{2} \\
& \dot{x}_{4}=-c x_{1} x_{3}+d x_{4}
\end{aligned}
$$

where $x_{i},(i=1,2,3,4)$ are the state variables and $a, b, c, d$ are unknown system parameters.

The system (13) is hyperchaotic when the parameter values are taken as

$$
a=0.4, \quad b=0.175, c=0.8 \text { and } d=0.01 \text {. }
$$

As the slave system, we consider the controlled hyperchaotic Newton-Leipnik dynamics described by

$$
\begin{aligned}
& \dot{y}_{1}=-a y_{1}+y_{2}+10 y_{2} y_{3}+y_{4}+u_{1} \\
& \dot{y}_{2}=-y_{1}-0.4 y_{2}+5 y_{1} y_{3}+u_{2} \\
& \dot{y}_{3}=b y_{3}-5 y_{1} y_{2}+u_{3} \\
& \dot{y}_{4}=-c y_{1} y_{3}+d y_{4}+u_{4}
\end{aligned}
$$

where $y_{i},(i=1,2,3,4)$ are the state variables and $u_{i},(i=1,2,3,4)$ are the nonlinear controllers to be designed.

The synchronization error is defined by

$$
e_{i}=y_{i}-x_{i}, \quad(i=1,2,3,4)
$$

Then the error dynamics is obtained as

$$
\begin{aligned}
& \dot{e}_{1}=-a e_{1}+e_{2}+10\left(y_{2} y_{3}-x_{2} x_{3}\right)+e_{4}+u_{1} \\
& \dot{e}_{2}=-e_{1}-0.4 e_{2}+5\left(y_{1} y_{3}-x_{1} x_{3}\right)+u_{2} \\
& \dot{e}_{3}=b e_{3}-5\left(y_{1} y_{2}-x_{1} x_{2}\right)+u_{3} \\
& \dot{e}_{4}=-c\left(y_{1} y_{3}-x_{1} x_{3}\right)+d e_{4}+u_{4}
\end{aligned}
$$


International Journal of Information Technology Convergence and Services (IJITCS) Vol.1, No.3, June 2011

Let us now define the adaptive control functions $u_{1}(t), u_{2}(t), u_{3}(t), u_{4}(t)$ as

$$
\begin{aligned}
& u_{1}=\hat{a} e_{1}-e_{2}-10\left(y_{2} y_{3}-x_{2} x_{3}\right)-e_{4}-k_{1} e_{1} \\
& u_{2}=e_{1}+0.4 e_{2}-5\left(y_{1} y_{3}-x_{1} x_{3}\right)-k_{2} e_{2} \\
& u_{3}=-\hat{b} e_{3}+5\left(y_{1} y_{2}-x_{1} x_{2}\right)-k_{3} e_{3} \\
& u_{4}=\hat{c}\left(y_{1} y_{3}-x_{1} x_{3}\right)-\hat{d} e_{4}-k_{4} e_{4}
\end{aligned}
$$

where $\hat{a}, \hat{b}, \hat{c}, \hat{d}, \hat{\varepsilon}$ and $\hat{f}$ are estimates of the parameters $a, b, c, d, \varepsilon$ and $f$ respectively, and $k_{i},(i=1,2,3,4)$ are positive constants.

Substituting the control law (17) into (16), we obtain the error dynamics as

$$
\begin{aligned}
& \dot{e}_{1}=-(a-\hat{a}) e_{1}-k_{1} e_{1} \\
& \dot{e}_{2}=-k_{2} e_{2} \\
& \dot{e}_{3}=(b-\hat{b}) e_{3}-k_{3} e_{3} \\
& \dot{e}_{4}=-(c-\hat{c})\left(y_{1} y_{3}-x_{1} x_{3}\right)+(d-\hat{d}) e_{4}-k_{4} e_{4}
\end{aligned}
$$

Let us now define the parameter errors as

$$
e_{a}=a-\hat{a}, \quad e_{b}=b-\hat{b}, \quad e_{c}=c-\hat{c}, \quad e_{d}=d-\hat{d}
$$

Substituting (19) into (18), the error dynamics simplifies to

$$
\begin{aligned}
& \dot{e}_{1}=-e_{a} e_{1}-k_{1} e_{1} \\
& \dot{e}_{2}=-k_{2} e_{2} \\
& \dot{e}_{3}=e_{b} e_{3}-k_{3} e_{3} \\
& \dot{e}_{4}=-e_{c}\left(y_{1} y_{3}-x_{1} x_{3}\right)+e_{d} e_{4}-k_{4} e_{4}
\end{aligned}
$$

For the derivation of the update law for adjusting the estimates of the parameters, the Lyapunov approach is used.

Consider the quadratic Lyapunov function

$$
V\left(e_{1}, e_{2}, e_{3}, e_{4}, e_{a}, e_{b}, e_{c}, e_{d}\right)=\frac{1}{2}\left(e_{1}^{2}+e_{2}^{2}+e_{3}^{2}+e_{4}^{2}+e_{a}^{2}+e_{b}^{2}+e_{c}^{2}+e_{d}^{2}\right)
$$

which is a positive definite function on $R^{8}$.

Note also that

$$
\begin{array}{ll}
\dot{e}_{a}=-\dot{\hat{a}}, & \dot{e}_{b}=-\dot{\hat{b}} \\
\dot{e}_{c}=-\dot{\hat{c}}, & \dot{e}_{d}=-\dot{\hat{d}}
\end{array}
$$


Differentiating $V$ along the trajectories of (20) and using (22), we obtain

$$
\begin{aligned}
\dot{V}= & -k_{1} e_{1}^{2}-k_{2} e_{2}^{2}-k_{3} e_{3}^{2}-k_{4} e_{4}^{2}+e_{a}\left[-e_{1}^{2}-\dot{\hat{a}}\right]+e_{b}\left[e_{3}^{2}-\dot{\hat{b}}\right] \\
& +e_{c}\left[-e_{4}\left(y_{1} y_{3}-x_{1} x_{3}\right)-\dot{\hat{c}}\right]+e_{d}\left[e_{4}^{2}-\dot{\hat{d}}\right]
\end{aligned}
$$

In view of Eq. (23), the estimated parameters are updated by the following law:

$$
\begin{aligned}
& \dot{\hat{a}}=-e_{1}^{2}+k_{5} e_{a} \\
& \dot{\hat{b}}=e_{3}^{2}+k_{6} e_{b} \\
& \dot{\hat{c}}=-e_{4}\left(y_{1} y_{3}-x_{1} x_{3}\right)+k_{7} e_{c} \\
& \dot{\hat{d}}=e_{4}^{2}+k_{8} e_{d}
\end{aligned}
$$

where $k_{5}, k_{6}, k_{7}$ and $k_{8}$ are positive constants.

Substituting (24) into (23), we get

$$
\dot{V}=-k_{1} e_{1}^{2}-k_{2} e_{2}^{2}-k_{3} e_{3}^{2}-k_{4} e_{4}^{2}-k_{5} e_{a}^{2}-k_{6} e_{b}^{2}-k_{7} e_{c}^{2}-k_{8} e_{d}^{2},
$$

which is a negative definite function on $R^{8}$.

Thus, by Lyapunov stability theory [18], it is immediate that the synchronization error and the parameter error decay to zero exponentially with time for all initial conditions.

Hence, we have proved the following result.

Theorem 2. The identical hyperchaotic Newton-Leipnik systems (13) and (14) with unknown parameters are globally and exponentially synchronized for all initial conditions by the adaptive control law (17), where the update law for parameters is given by $(24)$ and $k_{i},(i=1, \ldots, 8)$ are positive constants.

\subsection{Numerical Results}

For the numerical simulations, the fourth order Runge-Kutta method is used to solve the two systems of differential equations (13) and (14) with the adaptive control law (17) and the parameter update law (24).

For the adaptive synchronization of the hyperchaotic Newton-Leipnik systems with parameter values

$$
a=0.4, \quad b=0.175, c=0.8 \text { and } d=0.01 \text {, }
$$

we apply the adaptive control law (17) and the parameter update law (24).

We take the positive constants $k_{i},(i=1, \ldots, 8)$ as $k_{i}=2$ for $i=1,2, \ldots, 8$. 
International Journal of Information Technology Convergence and Services (IJTTCS) Vol.1, No.3, June 2011

Suppose that the initial values of the estimated parameters are

$$
\hat{a}(0)=6, \hat{b}(0)=2, \hat{c}(0)=5 \text { and } \hat{f}(0)=5 \text {. }
$$

We take the initial values of the master system $(13)$ as $x(0)=(15,14,28,30)$.

We take the initial values of the slave system (14) as $y(0)=(27,10,20,16)$.

Figure 4 shows the adaptive chaos synchronization of the identical hyperchaotic NewtonLeipnik systems. Figure 5 shows that the estimated values of the parameters $\hat{a}, \hat{b}, \hat{c}$ and $\hat{d}$ converge to the system parameters $a=0.4, b=0.175, c=0.8$ and $d=0.01$.
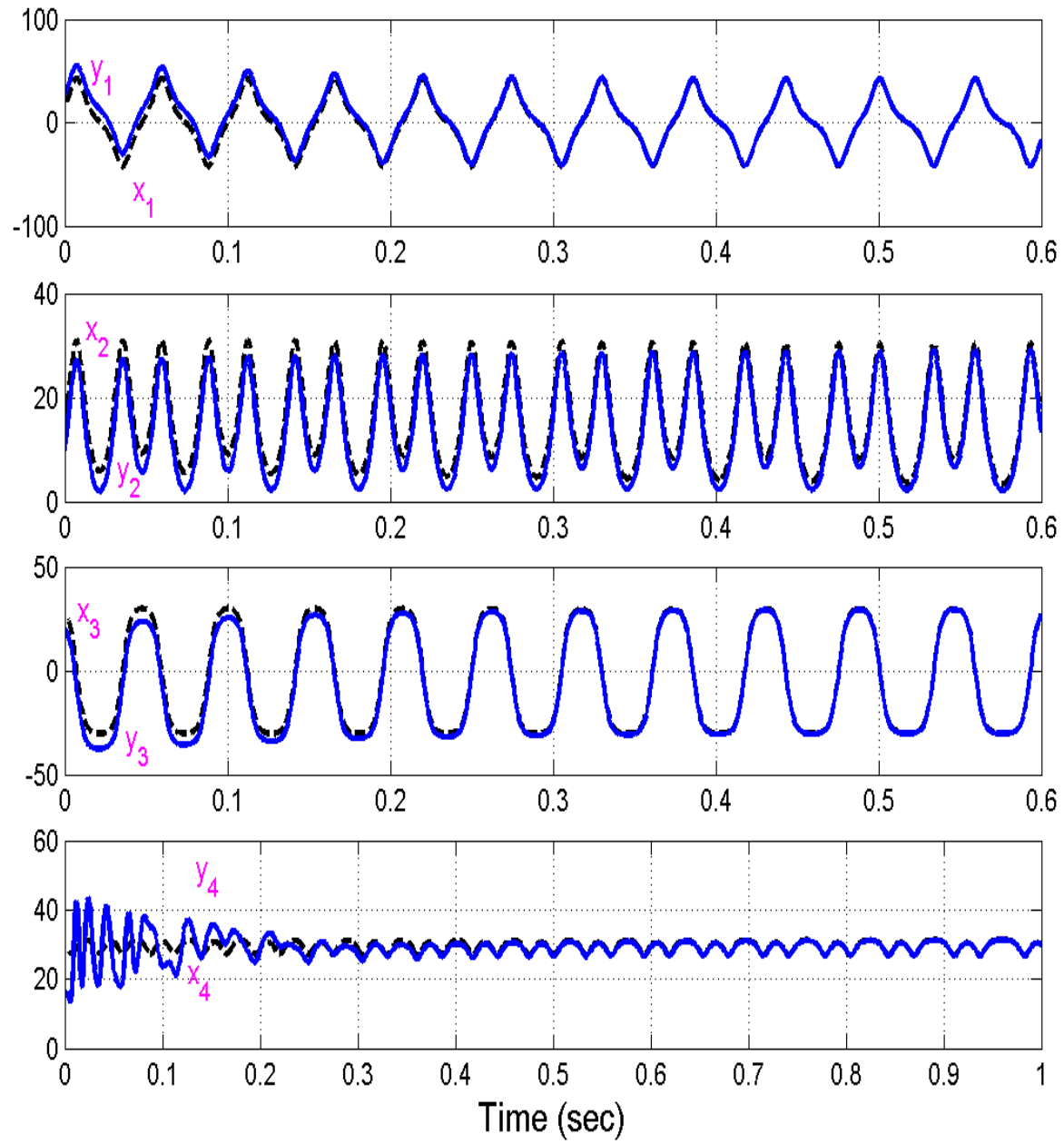

Figure 4. Adaptive Synchronization of the Identical Hyperchaotic Newton-Leipnik Systems 


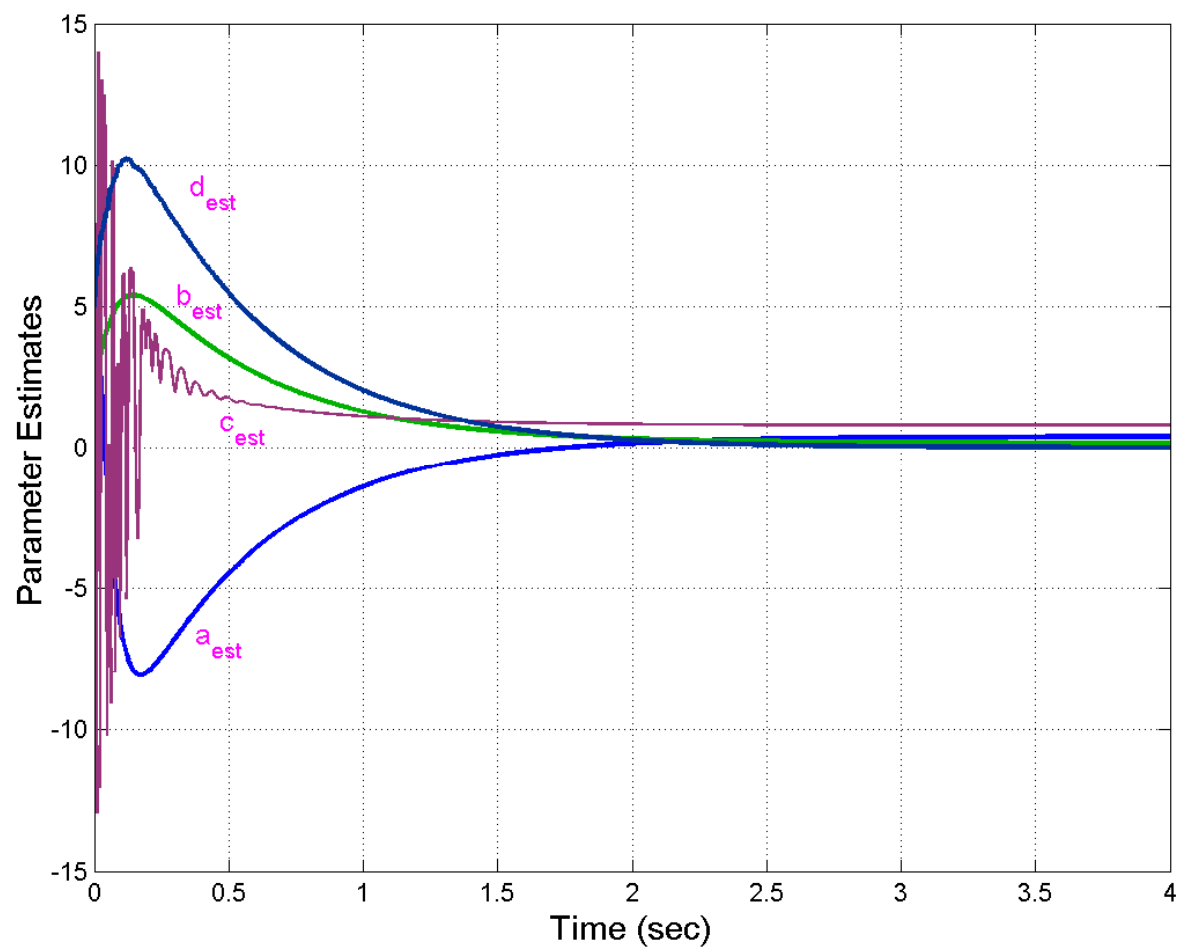

Figure 5. Parameter Estimates $\hat{a}(t), \hat{b}(t), \hat{c}(t), \hat{d}(t)$

\section{CONCLUSIONS}

In this paper, we applied adaptive control theory for the stabilization and synchronization of the hyperchaotic Newton-Leipnik system (Ghosh and Bhattacharya, 2010) with unknown system parameters. First, we designed adaptive control laws to stabilize the hyperchaotic NewtonLeipnik system to its unstable equilibrium point at the origin based on the adaptive control theory and Lyapunov stability theory. Then we derived adaptive synchronization scheme and update law for the estimation of system parameters for identical hyperchaotic Newton-Leipnik systems with unknown parameters. Our synchronization schemes were established using Lyapunov stability theory. Since the Lyapunov exponents are not required for these calculations, the proposed adaptive control method is very effective and convenient to achieve chaos control and synchronization of the hyperchaotic Newton-Leipnik system. Numerical simulations are shown to demonstrate the effectiveness of the proposed adaptive stabilization and synchronization schemes.

\section{REFERENCES}

[1] Alligood, K.T., Sauer, T. \& Yorke, J.A. (1997) Chaos: An Introduction to Dynamical Systems, Springer, New York.

[2] Ge, S.S., Wang, C. \& Lee, T.H. (2000) "Adaptive backstepping control of a class of chaotic systems,” Internat. J. Bifur. Chaos, Vol. 10, pp 1149-1156.

[3] Wang, X., Tian, L. \& Yu, L. (2006) "Adaptive control and slow manifold analysis of a new chaotic system,” Internat. J. Nonlinear Science, Vol. 21, pp 43-49. 
International Journal of Information Technology Convergence and Services (IJITCS) Vol.1, No.3, June 2011

[4] Sun, M., Tian, L., Jiang, S. \& Xun, J. (2007) "Feedback control and adaptive control of the energy resource chaotic system," Chaos, Solitons \& Fractals, Vol. 32, pp 168-180.

[5] Pecora, L.M. \& Carroll, T.L. (1990) "Synchronization in chaotic systems", Phys. Rev. Lett., Vol. 64, pp 821-824.

[6] Lakshmanan, M. \& Murali, K. (1996) Nonlinear Oscillators: Controlling and Synchronization, World Scientific, Singapore.

[7] Han, S.K., Kerrer, C. \& Kuramoto, Y. (1995) "Dephasing and bursting in coupled neural oscillators”, Phys. Rev. Lett., Vol. 75, pp 3190-3193.

[8] Blasius, B., Huppert, A. \& Stone, L. (1999) "Complex dynamics and phase synchronization in spatially extended ecological system”, Nature, Vol. 399, pp 354-359.

[9] Feki, M. (2003) "An adaptive chaos synchronization scheme applied to secure communication", Chaos, Solitons and Fractals, Vol. 18, pp 141-148.

[10] Murali, K. \& Lakshmanan, M. (1998) "Secure communication using a compound signal from generalized synchronizable chaotic systems", Phys. Rev. Lett. A, Vol. 241, pp 303-310.

[11] Yang, T. \& Chua, L.O. (1999) "Control of chaos using sampled-data feedback control”, Internat. J. Bifurcat. Chaos, Vol. 9, pp 215-219.

[12] Ott, E., Grebogi, C. \& Yorke, J.A. (1990) "Controlling chaos", Phys. Rev. Lett., Vol. 64, pp 1196-1199.

[13] Park, J.H. \& Kwon, O.M. (2003) "A novel criterion for delayed feedback control of time-delay chaotic systems”, Chaos, Solitons and Fractals, Vol. 17, pp 709-716.

[14] Yu, Y.G. \& Zhang, S.C. (2006) “Adaptive backstepping synchronization of uncertain chaotic systems”, Chaos, Solitons and Fractals, Vol. 27, pp 1369-1375.

[15] Liao, T.L. \& Tsai, S.H. (2000) "Adaptive synchronization of chaotic systems and its applications to secure communications", Chaos, Solitons and Fractals, Vol. 11, pp 1387-1396.

[16] Konishi, K.., Hirai, M. \& Kokame, H. (1998) "Sliding mode control for a class of chaotic systems", Phys. Lett. A, Vol. 245, pp 511-517.

[17] Ghosh, D. \& Bhattacharya, S. (2010) "Projective synchronization of new hyperchaotic system with fully unknown parameters," Nonlinear Dynamics, Vol. 61, pp 11-21.

[18] Hahn, W. (1967) The Stability of Motion, Springer, New York.

\section{Author}

Dr. V. Sundarapandian is a Professor (Systems and Control Engineering), Research and Development Centre at Vel Tech Dr. RR \& Dr. SR Technical University, Chennai, India. His current research areas are: Linear and Nonlinear Control Systems, Chaos Theory, Dynamical Systems and Stability Theory, etc. He has published over 125 research articles in international journals and two text-books with Prentice-Hall of India, New Delhi, India. He has published over 45 papers in International Conferences and 90 papers in National Conferences. He has delivered several Key Note Lectures on Control Systems, Chaos Theory, Scientific Computing, SCILAB, etc.

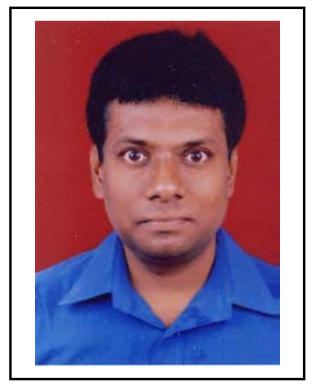

